\title{
Application of 3D Visualization Technology in Virtual Reality
}

\author{
JianLan Ren,Jianhong Ren \\ Jiangxi Vocational and Technical College of Communication; Jiangxi, Nanchang 330013, China \\ 111166@163.com
}

Keywords: Virtual Reality; 3D Visualization; Scene Modeling

\begin{abstract}
Virtual reality system allows users to start from their own point of view, and take advantage of natural skills and related equipment for the generation of virtual world objects to browse and interact. In virtual reality platform, 3D visualization technology plays a vital role. In this article, we carry out study on the key technologies of virtual modeling, and propose the virtual scene construction methods by 3D visualization technology, including vision modeling, entity modeling and environmental landscape modeling.
\end{abstract}

\section{Introduction}

With the rapid development of information technology in terms of computer and network, virtual reality technology has been widely used in many fields including scientific research, education, commercial, industrial, military, entertainment etc. Virtual reality technology uses the characteristics of computer and network technology to generate a realistic three-dimensional visual, auditory, tactile and even sense of smell, and so on. It allows users to start from their own point of view, and take advantage of natural skills and related equipment for the generation of virtual world objects to browse and interact. From the perspective of application range of virtual reality, the realm of virtual reality must be built on the basis of the design of the model, that is, the integrated application of three-dimensional modeling technology.

For virtual environments, three-dimensional visualization modeling technology will bring authenticity, sensuality and a variety of other visual effects, And under the development of different modeling tools and language technology, it could derive better solutions, especially for entity modeling and real-time interaction. Overall, application of three-dimensional visualization modeling technology would optimize the effective construction of virtual reality system, and promote the development of human thinking and creativity.

\section{Key preparation of Virtual Scene Modeling}

Construction of the virtual environment is the foundation of a virtual reality system, and virtual scene modeling is a key step. The virtual scene model not only needs to be realistic and beautiful, but also needs to meet the requirements of real-time systems.

Modeling Process Optimization. Quality of virtual scene modeling will directly affect the photorealistic and interactivity sense of the virtual reality platform. For the purpose of better implement virtual scenario planning, we carry out virtual scene modeling process optimization, for the perspective of structure optimization, model optimization and scene optimization, as shown in Figure: 


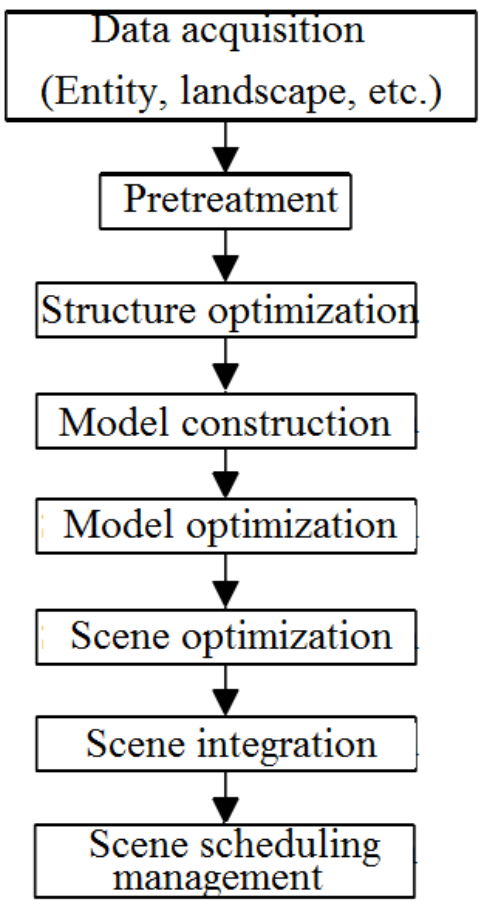

Fig. 1. Virtual Scene Model Process

The main steps to build three-dimensional virtual scene can be generalized as follow:

(1) Preparatory: data acquisition and pre-processing; determine the scenes and model structure, and conduct optimization.

(2) Scene construction: model construction, including natural landscape, environmental landscape and three-dimensional entity, etc.

(3) Finishing work: including integrated optimization of model, integrated optimization of scene and scheduling management.

Data Acquisition and Processing. The virtual scene is a reflection of the objective world, and scene construction is the digital process of the real environment. Therefore, before modeling, it requires to collect and process the corresponding data of real environment.

(1) Data acquisition

The accuracy of the data acquisition determines the accuracy of the model and the authenticity of the whole scene. The data needed in virtual reality platform includes model information, texture information and peripheral landscape information, etc. the natural landscape, physical appearance and geometric shape data is mainly from the floor plan, architectural drawings, planning drawings, photo and video files; texture information mainly comes from photographic processing.

According to the experience involved in the actual data collection, attention should be paid to the following aspects:

a) Acquisition order: in coordination with the layout of the entity in the scene, and name and number it;

b) Acquisition time: determine a fixed time for photography, so that ensure consistent photographic effects;

c) Acquisition backup: after the data is collected, it should be backed up into the system, in case of data loss.

(2) Data processing

Based on the requirements of virtual reality platform, we need to process the data collected, including the following types of data: 
a) Natural landscape data: natural landscape data includes the sky, clouds, sun and earth information involved in virtual scene. It only requires simple processing by image processing software.

b) Scene entity data: it mainly refers to dimension, structure and connection of three dimensional models in scene modeling. To acquire precise scene entity data, we should pay attention to the single entity, partial scene, whole scene and scale structure of various parts of the junction.

c) Texture data: texture data can be used in some surface texture characteristics entities and simple geometric model mapping. The texture data used in virtual mainly comes from photography on the spot.

\section{Virtual Scene Construction by 3D Visualization Technology}

Virtual scene construction is to achieve the specific geometric modeling of each entity and environmental landscape. The appearance of an object is determined by its surface texture, color, texture, light and other factors. Here we elaborate the 3D virtual scene construction method, from the perspective of vision modeling, entity modeling and environmental landscape modeling.

Vision Modeling. The common features of vision modeling are: when the viewpoint distance is very far away, the model itself requires no details, but only needs to emphasize the performance effect. We use three-dimensional modeling software 3dsmax to realize vision modeling. When the viewpoint moves in the space composed of ground, boundary facades, and coupled with appropriate lighting effect, it can bring intense depth sense of the vision. In order to increase the dynamic effect, we can also adopt texture transformation method to express the distant scene. For example, we employ hemisphere method to achieve the sky and cloud effect modeling, as shown in Fig. 2:

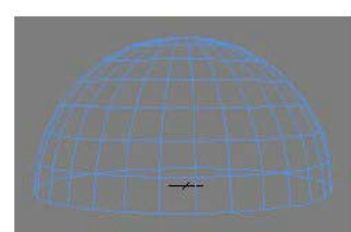

(a) Hemisphere sky wireframes

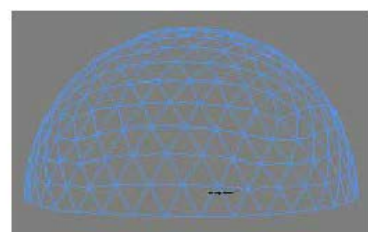

(b) GeoSphere sky wireframes

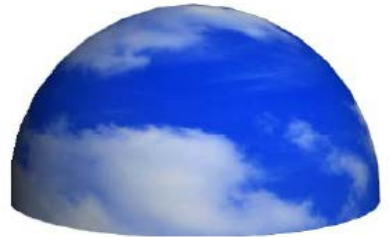

(c) Hemisphere sky renderings

Fig. 2. Hemisphere sky modeling

If we want to allow the sky to show the effect of various weathers, we can assign different materials and textures to the sky model in the real-time control stage.

Entity Modeling. Three-dimensional entity modeling in virtual scene mainly includes static entity modeling and dynamic entity modeling. The former refers to the static objects including buildings, trees, roads, mountains and so on; while the latter mainly refers to a variety of simulation entities with motion properties, including windmill, rivers, animals, machine and so on. In the virtual reality platform, the modeling of static entity mainly focus on appearance modeling, instead of internal details modeling.

Modeling of entity is mainly to use geometric modeling technology to simulate the entity in terms of shape and appearance, and meanwhile take advantage of texture mapping and other auxiliary technical means to reduce the complexity of the model. The modeling sequence is determined according to the position and importance of the static entity, and then carries out a separate modeling of each important static entity in turn.

The general steps of entity modeling are as follow:

a) Collect modeling data; 
b) Determine the hierarchical structure of the model;

c) Construct the model;

d) Delete redundant geometric elements;

e) Calculate the shadow;

f) Utilize texture mapping.

Environmental Landscape Modeling. Environmental landscape mainly includes grass, trees, flowers, lights and hedges, etc. Decoration of environmental landscape could greatly enhance the scene realism and fidelity. However, due to the limitation of modeling tools, the modeling of irregular objects has always been a hot and difficult research topic. Taking grass and trees for example, we use simple geometry to perform their appearance, and then show the authenticity by texture mapping.

(1) Grass

With 3dsmax, the specific modeling steps of grass landscape are as follow:

a) Reference to the scenario plan provided, to determine the location and contour grass from the global perspective;

b) Determining an edge region and the central area of grassland, and complete modeling;

c) Conduct texture mapping on the edge areas and central area.

The modeling effect of grassland is shown in Fig. 3:
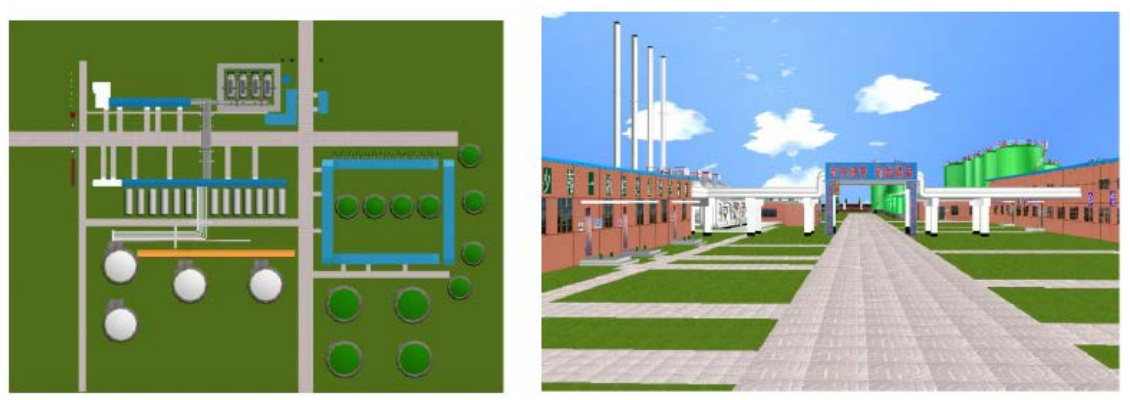

Fig. 3. Modeling effect of grassland

(2) Trees

Generally, the more green vegetation, the better visual effect will be, so tree modeling is also an important part of virtual reality platform. Due to the irregular shape of the trees, it is difficult to use basic geometry to represent a tree as entity modeling. Therefore, it is not feasible to apply detail modeling method to describe a large number of trees. In general, cross intersect method by 3dsmax is the most commonly used.

Cross intersect method is to use two cross plane to construct tree model. In two mutually perpendicular planes, mapped with the same texture of trees, people can always see the same image of trees from different angles, because of human visual difference. This method can not only reduce the amount of calculation, but also improve the display speed. The modeling effect by Cross intersect method is shown in Fig. 4: 


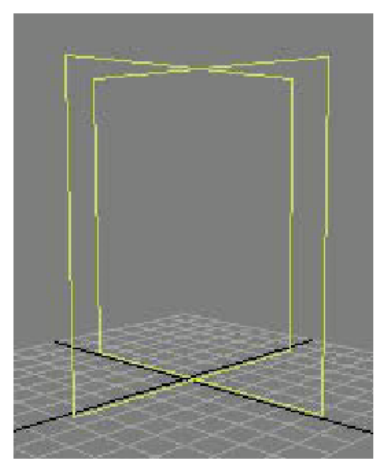

(a) Create two perpendicular plane

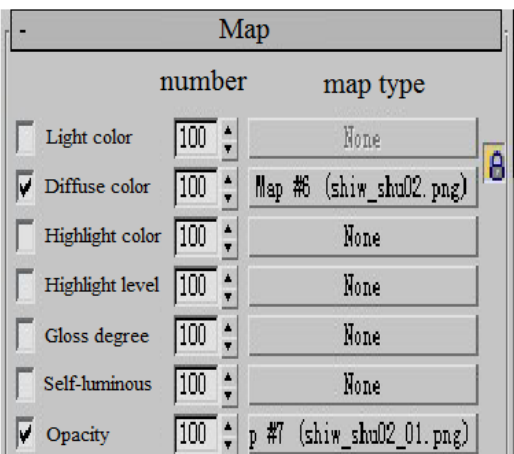

(b) set the texture parameters

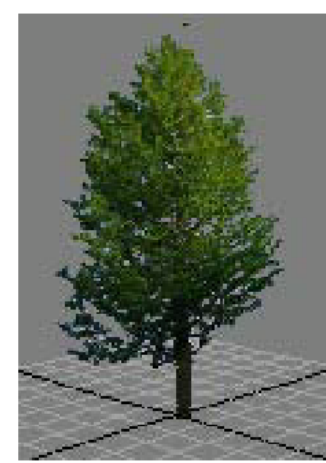

(c) Generate the tree model

Fig. 4. Modeling effect of tree by cross intersect method

The construction process is: firstly, create two perpendicular plane in view (as Fig. 4 (a)), then set the texture parameters in the material editor (as Fig. 4 (b)), that is, select different textures in the diffuse and opacity maps, respectively, and finally assign this material to the cross plane and generate the tree model (as Fig. 4 (c)).

\section{Conclusion}

Taking advantage of the three-dimensional modeling software 3dsmax and its control engine Virtools, we demonstrate the construction of 3D virtual reality scene and the modeling technology, and the modeling effect could well meet the requirements of large scale, real-time, interactivity and authenticity. Separately, in the virtual scene construction, we introduce the modeling method of vision view, entity, and environmental landscape, and provide effective solutions and summarize for further research on application of 3D visualization technology and virtual reality platform.

\section{References}

[1] Akpan I J. Virtual Reality, 3D Visualization and Simulation in Decision Support[C]// International Conference on Computer Science and Information Systems. 2009.

[2] Ma M, Zheng H, Lallie H. Virtual Reality and 3D Animation in Forensic Visualization *[J]. Journal of Forensic Sciences, 2010, 55(5):1227-31.

[3] Cheng S, Chen G, Chen Q, et al. Research on 3D dynamic visualization simulation system of toxic gas diffusion based on virtual reality technology[J]. Process Safety \& Environmental Protection, 2009, 87(3):175-183.

[4] Lin Y E, Qiu L H. Research of virtual reality 3D visualization of mechanism design on web[J]. Machinery Design \& Manufacture, 2006.

[5] Cui Q, Sun W, Wang R, et al. Applied study of 3D visualization the finite element analysis based on virtual reality technology[J]. Modern Manufacturing Engineering, 2015.

[6] Herrmann H, Pastorelli E. Virtual Reality Visualization for Photogrammetric 3D Reconstructions of Cultural Heritage[M]// Augmented and Virtual Reality. Springer International Publishing, 2014:283-295. 